\title{
Hypoxia induces differential expression patterns of osteopontin and CD44 in colorectal carcinoma
}

\author{
GISELA WOHLLEBEN ${ }^{1}$, KONSTANTIN HAUFF ${ }^{1}$, MARTIN GASSER $^{2}$, ANA MARIA WAAGA-GASSER $^{2,3}$, \\ TANJA GRIMMIG $^{2}$, MICHAEL FLENTJE ${ }^{1}$ and BÜLENT POLAT ${ }^{1}$ \\ Departments of ${ }^{1}$ Radiation Oncology, and ${ }^{2}$ Surgery I, Molecular Oncology and Immunology, University of Würzburg, \\ D-97080 Würzburg, Germany; ${ }^{3}$ Transplantation Research Center, Brigham and Women's Hospital, \\ Harvard Medical School, Boston, MA 02115, USA
}

Received May 26, 2017; Accepted October 4, 2017

DOI: $10.3892 /$ or.2017.6068

\begin{abstract}
The plasma protein osteopontin (OPN) is considered to be a tumor biomarker, where elevated plasma levels are associated with poor prognosis. Additionally, OPN is expressed in the presence of tumor hypoxia, which is an adverse prognostic factor in radiation oncology. One of its receptors, the proposed tumor stem cell marker CD44, is also associated with aggressive tumors, shown for example in colon cancer. The expression of CD44 and its splice variants (particularly CD44v6) can be upregulated by OPN itself. In the present study, we aimed to investigate the influence of hypoxia on the expression of OPN and its binding partners CD44 and CD44v6 in colon carcinoma cell lines in vitro, using SW480, SW620, HT29 and HCT116 cells. Additionally, we investigated the effect of irradiation on the expression pattern of OPN and its ligands, and the influence of hypoxia on the clonogenic survival of the cells after irradiation. While the expression patterns were nearly unaltered by irradiation, hypoxia led to an upregulation of OPN protein expression and an increase in the radioresistance in all tested colorectal carcinoma cell lines. However, a similar clear statement with regard to the expression of CD44 and CD44v6 is not possible. We hypothesize that the OPN receptors differ in their expression pattern between cell lines depending on the degree of their malignancy.
\end{abstract}

\section{Introduction}

Osteopontin (OPN) is a secreted extracellular matrix protein, which can be detected in tissues, serum and urine under physiological conditions. Due to an increased expression in many tumor tissues, increased serum levels in cancer patients are

Correspondence to: Dr Bülent Polat, Department of Radiation Oncology, University Hospital Würzburg, Josef-SchneiderStrasse 11, D-97080 Würzburg, Germany

E-mail: polat_b@ukw.de

Key words: osteopontin, CD44s, CD44v6, hypoxia, colorectal cancer considered as a negative prognostic tumor biomarker. Various studies have shown that elevated OPN levels are associated with tumor invasion and metastasis particularly in head and neck tumors, lung, breast, prostate and colorectal cancer, resulting in a worse prognosis of the disease (1-3).

Furthermore, increased levels of OPN could be detected in tumors with reduced oxygen content (tumor hypoxia) (4-6). In addition, tumor hypoxia is associated with decreased radiation sensitivity and a poor prognosis after radiation therapy (7-9). Furthermore, we and others recently showed, that silencing of OPN expression caused an increase in radiosensitivity (10-12).

One of the binding partners of OPN is the CD44 (CD44s) receptor and some of its isoforms (13). These are expressed in numerous tumor types and can be upregulated by OPN itself as shown in stomach, liver, thyroid and breast cancer tissues (14). Via CD44, signaling pathways are activated, which regulate cell proliferation, migration and survival signals and lead to metastasis (12). Recently, it was shown in a mouse model that a CD44 antagonist delayed the tumor growth of glioblastoma cells (15). By interaction of OPN with CD44 and its various splice variants, the PLC- $\gamma$-dependent Akt pathway is activated which contributes to the motility and survival of tumor cells (16). Although there are many splice variants of CD44, particularly variant $6(\mathrm{CD} 44 \mathrm{v} 6)$ is highly expressed along with OPN in many cancers such as breast and gastric cancer or leukemia and is considered a marker for advanced tumor disease (17-22).

With this in mind, we analyzed the effect of hypoxia on the expression of OPN and its receptors CD44s and CD44v6 in 4 different human colorectal carcinoma cell lines (SW480, SW620, HT29 and HCT116). We aimed to ascertain whether hypoxic conditions lead to an increase in OPN and in parallel to an upregulation of CD44s, particularly of CD44v6.

\section{Materials and methods}

Cell culture. The human colon adenocarcinoma cell lines SW480, SW620 (this cell line was isolated from a lymph node metastasis of the same patient from which the SW480 cell line is derived), HT29 and HCT116 were purchased from the American Type Culture Collection (ATCC; Manassas, VA, USA). 
SW480 and SW620 cells were grown in RPMI medium supplemented with $10 \%$ fetal bovine serum (PAA, Cölbe Germany), $2 \mathrm{mML}$-glutamine and penicillin (100 IU $/ \mathrm{ml}) /$ streptomycin $(100 \mu \mathrm{g} / \mathrm{ml})$ and $10 \%$ non-essential amino acids and pyruvat. HT29 and HCT116 cells were cultured in Dulbecco's modified Eagle's medium (DMEM) supplemented with $10 \%$ fetal bovine serum, $2 \mathrm{mM}$ L-glutamine and penicillin $(100 \mathrm{IU} / \mathrm{ml}) / \mathrm{streptomycin}(100 \mu \mathrm{g} / \mathrm{ml})$ in a humidified atmosphere of $5 \% \mathrm{CO}_{2}$ at $37^{\circ} \mathrm{C}$.

To compare expression of OPN, CD44s and C44v6 under normoxic and hypoxic conditions at the mRNA and protein level, the cells were seeded in standard $75 \mathrm{~cm}^{2}$ tissue culture flasks (Greiner Bio-One, Frickenhausen, Germany) at a concentration of $1 \times 10^{6} /$ flask. Afterwards, the cells were allowed to adhere. While a portion of cells was kept under normoxic conditions $\left(21 \% \mathrm{O}_{2}, 5 \% \mathrm{CO}_{2}\right.$ at $\left.37^{\circ} \mathrm{C}\right)$, the other portion was transferred into a hypoxic glove box in parallel (Coy Laboratory Products, Grass Lake, MI, USA) and cultured at $0.1 \% \mathrm{O}_{2}\left(5 \% \mathrm{CO}_{2}\right.$ at $\left.37^{\circ} \mathrm{C}\right)$ for $48 \mathrm{~h}$. Afterwards, the cells were harvested for preparing RNA or whole-cell lysates.

To examine the effect of additional irradiation on the expression of OPN and its ligands (CD44 and CD44 v6) under the conditions already mentioned, a portion of the cells was irradiated $24 \mathrm{~h}$ after onset of the cultures at doses of 2 and 8 Gy using a 6-MV linear accelerator (Siemens, Concord, CA, USA) at a dose rate of $2 \mathrm{~Gy} / \mathrm{min}$. After another $24 \mathrm{~h}$ of growth under standard or hypoxic conditions, respectively, the cells were analyzed accordingly. In the case of the colony forming assay, the cells were treated in the same way but were irradiated at doses of 2,3, 5, 7 and 8 Gy or left untreated.

RNA isolation and real-time quantitative PCR (RT-qPCR). Total RNA of the cells was purified with the RNeasy Mini ${ }^{\circledR}$ kit following the instructions given by the supplier (Qiagen, Hilden, Germany). The subsequent reverse transcription of the RNA into cDNA was carried out using First Strand cDNA Synthesis kit as recommended by the manufacturer (Fermentas GmbH, St. Leon-Rot, Germany). Afterwards quantitative real-time PCR was performed to amplify cDNA coding for OPN, CD44s and the housekeeping gene hypoxanthine phosphoribosyltransferase (HPRT) using TaqMan ${ }^{\circledR}$ Gene Expression Assays (Applied Biosystems, Foster City, CA, USA). CAIX RT-qPCR analysis was performed in the same way to confirm the hypoxic culture conditions. As the threshold cycle $(\mathrm{Ct})$ the number of cycles required to cross the threshold was used. The crossing point of the housekeeping gene HPRT was taken to normalize the amount of cDNA in each sample. Changes in the expression were detected and quantified using the comparative $\mathrm{Ct}$ method $\left(2^{-\Delta \Delta \mathrm{Cq}}\right)(23)$.

Western blot analyses. Whole-cell lysates were prepared by lysing the cells in RIPA buffer according to standard protocols. The proteins were separated by electrophoresis according to their size by running on 4-12\% Bis-Tris gradient gels (Invitrogen, Karlsruhe, Germany). Afterwards they were transferred to polyacrylamide membranes (Invitrogen) and analyzed by western blotting. Polyclonal rabbit anti-human-OPN FL-314 and monoclonal mouse anti-human-HPRT antibodies were purchased from Santa Cruz Biotechnology, Inc. (Heidelberg, Germany). Mouse monoclonal anti-CD44std (BMS 150) and mouse monoclonal anti-CD44v6 (BMS 125) antibodies were purchased from eBioscience (Vienna, Austria). The mouse monoclonal antibody against CAIX is a product of BioScience (Bratislava, Slovakia). Detection was achieved using speciesspecific horseradish peroxidase-coupled secondary antibodies (Dako, Hamburg, Germany) and Amersham ${ }^{\mathrm{TM}}$ ECL $^{\mathrm{TM}}$ Select Western Blotting detection reagent (GE Healthcare, Chalfont St. Giles, Buckinghamshire, UK).

ELISA assays. For detection of OPN, CD44s and CD44v6 protein levels in the non-concentrated supernatants of the different cell lines, cultured under normoxic or hypoxic conditions, enzyme-linked immunosorbent assay (ELISA) kits from IBL (Immuno-Biological Laboratories Co., Ltd., Gunma, Japan) were used and performed according to the manufacturer's instructions.

Flow cytometric analysis. To detect OPN in the cells, the cells were fixed in ice-cold ethanol and permeabilized with $0.5 \%$ Triton $\mathrm{X}$ for $10 \mathrm{~min}$. To block unspecific protein-protein interactions, the cells were incubated with $5 \%$ FCS for $1 \mathrm{~h}$ at $+4^{\circ} \mathrm{C}$. Then, an anti-OPN antibody from Abcam (ab8448; Cambridge, UK) was added overnight $\left(a t+4^{\circ} \mathrm{C}\right)$. For the secondary antibody, Alexa Fluor 488 goat anti-rabbit (H+L) (Invitrogen) was used at a dilution of $1 / 1,000$ for $1 \mathrm{~h}$. The analyses were performed with a FACScan ${ }^{\mathrm{TM}}$ (Becton-Dickinson, Mountain View, CA, USA). The output data, presented as geometrical means, were analyzed using the Flowing Software obtained from P. Terho (Turku Centre for Biotechnology, Turku, Finland).

Clonogenic survival assay. Standard colony formation assays were performed, analyzing the survival of cells after irradiation by graded single doses ( $0-8 \mathrm{~Gy}$ ). For that, different numbers of cells were seeded in 6-well plates, depending on the radiation dose previously defined, and were incubated under normoxic $\left(21 \% \mathrm{O}_{2}, 5 \% \mathrm{CO}_{2}\right.$ at $\left.37^{\circ} \mathrm{C}\right)$ or hypoxic $(0.1 \%$ $\mathrm{O}_{2}, 5 \% \mathrm{CO}_{2}$ at $37^{\circ} \mathrm{C}$ ) conditions for 2 weeks. For each exposure point, 3 replicates were carried out. Furthermore, the colony survival assay was carried out 3 times for each cell line. For visualization of the colony formation (colonies $\geq 50$ cells), cells were fixed and stained with crystal violet $(0.6 \%)$. The mean survival data for each individual cell line were fitted to the linear quadratic (LQ) model SF $=\exp \left(-\alpha X-\beta X^{2}\right)$, where $S F$ is the survival fraction, $X$ is the irradiation dose, and $\alpha$ and $\beta$ are the fitted parameters.

Statistical analysis. All experiments were performed in triplicates and repeated for at least 2 times. For PCR data, changes in expression levels were considered as significant when there was a minimum of a 2 -fold change. Flow cytometric data were quantified using the geometric mean. Fittings of experimental curves from the survival assays were carried out with Origin software (Microcal, Northampton, MA, USA).

\section{Results}

Expression pattern of OPN and CD44s at the mRNA level under hypoxia. Four different colorectal carcinoma cell lines (SW480, HT29, HCT116 and SW620) were grown under normoxic $\left(21 \% \mathrm{O}_{2}\right)$ or hypoxic $\left(0.1 \% \mathrm{O}_{2}\right)$ conditions. The 
A

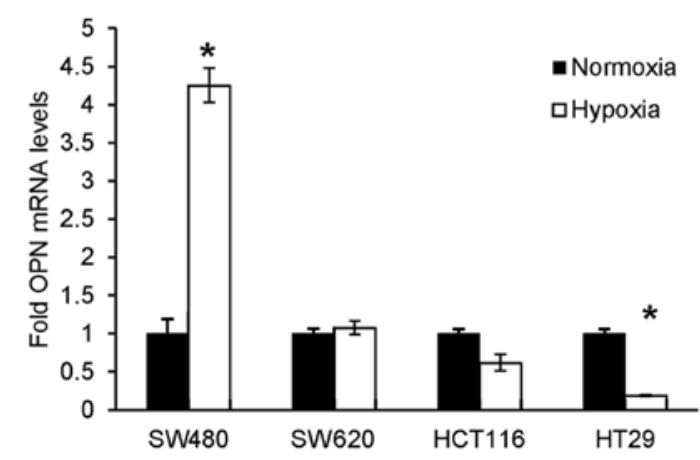

B

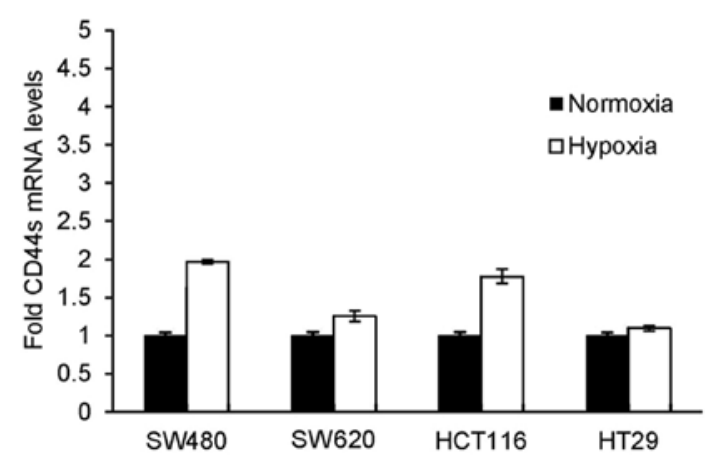

Figure 1. Quantitative RT-qPCR assays were performed to detect the mRNA levels of (A) OPN and (B) CD44s under normoxic and hypoxic conditions in SW480, SW620, HCT116 and HT29 colorectal carcinoma cell lines, respectively. Data represent the mean \pm SEM of 3 independent experiments. *A $\geq 2$-fold increase or decrease of $\leq 0.5$-fold compared to normoxic conditions, respectively.

effect of these parameters on CD44v6 mRNA expression was not possible, since TaqMan probes were not available for this splice variant of CD44. Therefore, CD44v6 expression was examined at the protein level.

To assess the effects of hypoxia on OPN and its receptor CD44s at the mRNA level, quantitative RT-PCR (RT-qPCR) was performed. SW480 cells showed a significant increase in OPN mRNA expression under hypoxic conditions (Fig. 1A). Concordant results were obtained concerning CD44s mRNA expression, but did not reach significance (Fig. 1B).
In contrast to SW480 cells, hypoxia caused a significant decrease in the OPN mRNA level in the HT29 cells and showed the same trend in the HCT116 cells, but without significance (Fig. 1A). Additionally, both cell lines showed contrary results with slightly increasing CD44s mRNA expression under hypoxic conditions. This observation was more pronounced in the HCT116 cells than in the HT29 cells (Fig. 1B).

To verify the hypoxic culture conditions, RT-qPCR was performed for CAIX expression, which is upregulated under hypoxia, depending on HIF-1 $\alpha$. All cell lines responded to hypoxia with a clear increase in CAIX expression, which was statistically significant (data not shown). SW480 cells showed a 73-fold and HCT116 a 75-fold increase in CAIX levels, respectively. The increase in CAIX mRNA expression in the HT29 (22-fold) and SW620 cells (27-fold) was not as strong, since both cell lines showed a background expression of CAIX (see also Fig. 2).

Expression pattern of $O P N, C D 44 s$ and $C D 44 v 6$ at the protein level under hypoxia. The influence of the different culture conditions on the expression of OPN, CD44 and CD44v6 at the protein level was tested by western blot analysis.

In all cell lines cultured under hypoxic conditions, there was an increase in OPN levels compared with the normoxic controls (Fig. 2). This increase was more pronounced in the SW480, HCT116 and HT29 cells than in the SW620 cells.

Expression of CD44s was slightly enhanced by hypoxia in the SW480 cells. In the SW620 cells there was nearly no difference in the CD44s protein levels observed depending on the culture conditions. HT29 cells responded to hypoxic conditions with a significant downregulation of CD44s protein, while it was upregulated in the HCT116 cells (Fig. 2). Protein levels of CD44v6, which is one of the CD44 isoforms associated with an increase in tumor malignancy, were unaffected by hypoxia in the cell lines SW480, SW620 and HCT116 (Fig. 2). Only in HT29 cells, hypoxic conditions led to a clear decrease in the CD44v6 protein level, comparable to the downregulation of CD44s in the same cell line.

In all cell lines, a significant upregulation of CAIX protein expression was observed under hypoxia, which confirmed cultivation under appropriate conditions. While there was no expression of CAIX protein detectable under normoxic conditions in the SW480 and HCT116 cells, HT29 and SW620 cells showed a weak 'background' secretion under normoxia

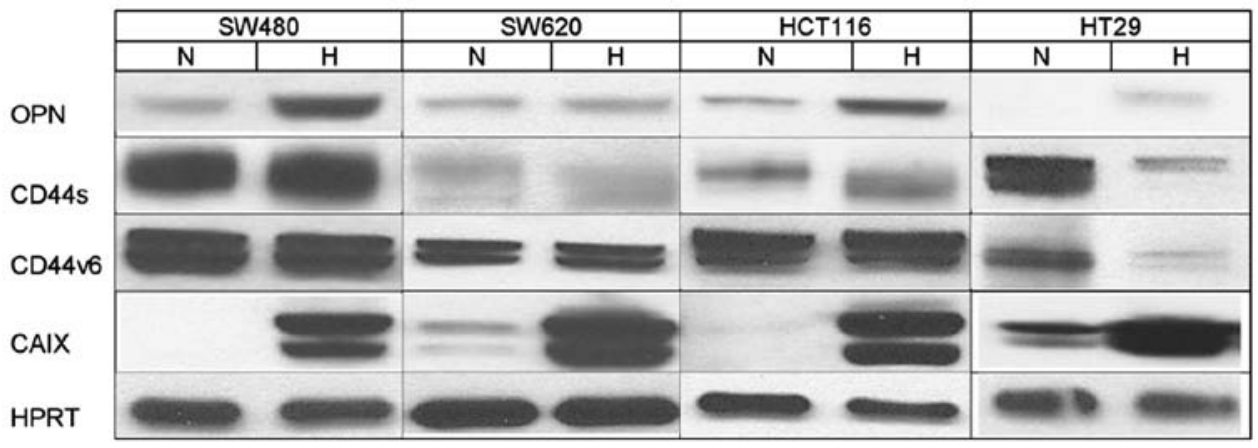

Figure 2. Western blots were run to detect the protein levels of OPN, CD44s, CD44v6 and CAIX expression under the influence of the different culture conditions (N, normoxia, H, hypoxia) in SW480, SW620, HCT116 and HT29 colorectal carcinoma cell lines. HPRT served as a loading control. 
A
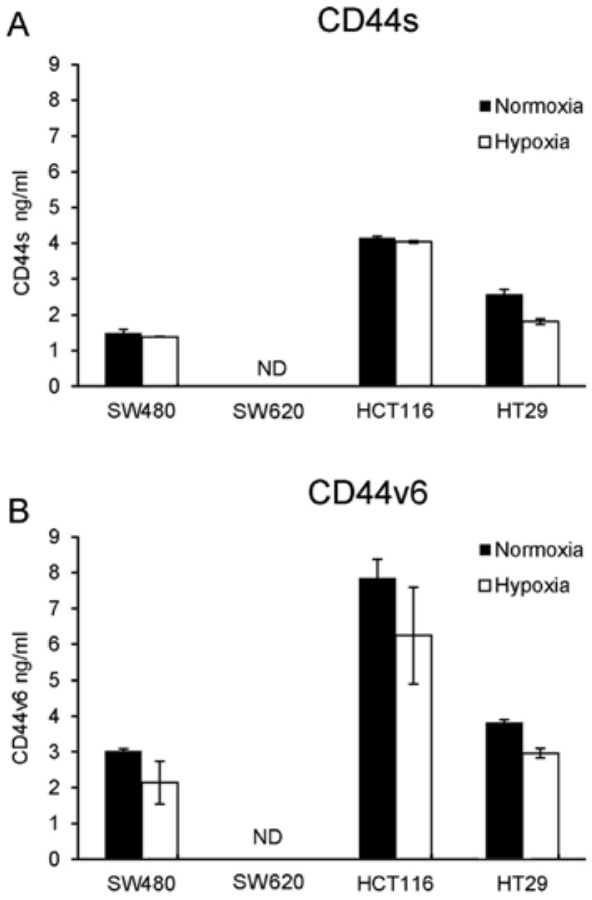

Figure 3. To detect the influence of normoxia and hypoxia on secretion of CD44s and CD44var.6, supernatants of the different colorectal carcinoma cell lines were tested by ELISA. Data showing CD44s and CD44var.6 concentrations in the different cell lines. No secretion at all was detectable (ND) in supernatants of SW620 cells.

as already observed at the mRNA level. HPRT was used as a loading control, respectively.

Influence of hypoxia on extracellular secretion of $O P N$, $C D 44$ and $C D 44 v 6$. Next, the effect of the different culture conditions on secretion of OPN, CD44s and CD44 v6 was investigated. Therefore cell culture supernatants were tested by a commercial ELISA system without further preparation (e.g. concentration) of the supernatants. In all cell lines, secretion of OPN was not detectable. Furthermore, neither CD44s nor CD44v6 was actively secreted by SW620 cells (Fig. 3A and B). All other cell lines showed secretion of both OPN receptors (Fig. 3). In contrast to HT29 cells, which showed reduced CD44s secretion under hypoxic conditions, SW480 and HCT116 cells showed nearly no reduction in CD44s secretion under hypoxia (Fig. 3A). In terms of CD44v6, hypoxia caused a decrease in secretion in all cell lines (Fig. 3B).

Influence of irradiation on expression of $O P N$ and its ligands $C D 44$ and $C D 44 v 6$. An effect of irradiation on OPN mRNA expression was only observed in the SW480 cells grown under standard conditions (normoxia). While doses of $2 \mathrm{~Gy}$ induced only a slight increase, irradiation with 8 Gy led to a more pronounced OPN mRNA expression (data not shown). This was not the case for CD44 mRNA expression, neither in this nor in the other cell lines tested. Furthermore, there was also no influence of irradiation on protein expression and secretion of OPN and its ligands CD44 and CD44v6.

Intracellular immunofluorescence (IF) staining. Although the amount of OPN protein increased under hypoxia, no OPN

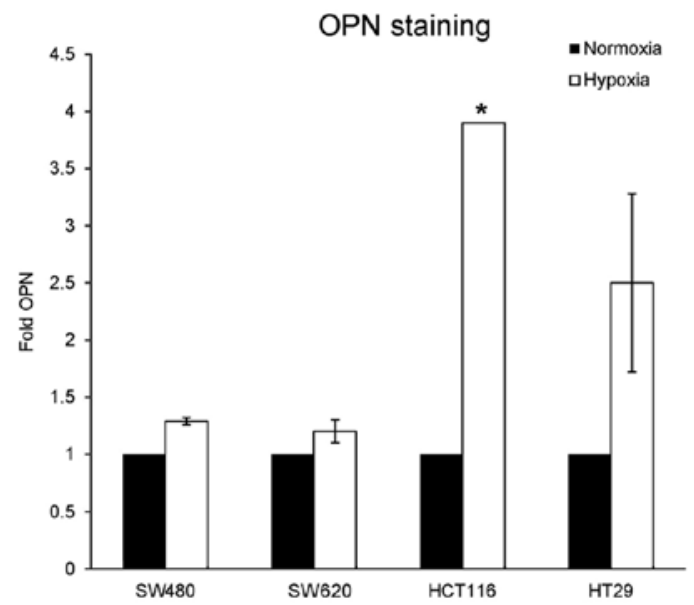

Figure 4. Immunofluorescence assays were performed to detect the intracellular OPN expression in SW480, SW620, HCT116 and HT29 colorectal carcinoma cell lines under different culture conditions. Shown are the results of the intracellular staining, evaluated by FACS analysis. The values of the cells cultured under normoxic conditions were normalized to 1 . Values are shown in geometrical means. " $\mathrm{A} \geq 2$-fold increase compared to normoxic conditions.

secretion was detectable in the supernatants of all cell lines tested by ELISA. Therefore intracellular FACS staining for OPN was performed to evaluate, whether OPN was stored intracellularly instead of being secreted into the cell culture medium. By flow cytometry, positive staining for OPN was observed in all cell lines, which was more pronounced when cells were cultured under hypoxia, particularly in HCT116 and HT29 cells (Fig. 4). These observations are in line with the results from the western blot analysis.

Effect of hypoxia and irradiation on radiosensitivity and clonogenic survival. To assess the effects of normoxia and hypoxia on radiosensitivity and survival, cell lines were cultured under standard $\left(21 \% \mathrm{O}_{2}\right)$ and hypoxic $\left(0.1 \% \mathrm{O}_{2}\right)$ conditions before they were exposed to different increasing irradiation doses up to $8 \mathrm{~Gy}$. Afterwards, the survival curves of the colony formation assays were calculated according to the LQ model. While the plating efficiency in SW480 cells was nearly not affected by the different culture conditions, all the other cell lines (SW620, HT29 and HCT116) showed a significant decrease in this parameter under hypoxia (Table I). An increase in clonogenic survival (SF2) was observed under oxygen reduction in all cell lines and ranged from $5 \%$ (HCT116), 6\% (HT29) and 7\% (SW480) up to 8\% in SW620 cells. The radiation dose yielding $10 \%$ survival (D10) showed a significant increase indicating radioresistance under hypoxia, which was most pronounced in the SW480 and HT29 cells. In the present study, radiation doses of 25 and $19.2 \mathrm{~Gy}$ were necessary to yield $10 \%$ survival, respectively. The corresponding survival curves are shown in Fig. 5.

\section{Discussion}

The presence of tumor hypoxia is an established adverse prognostic factor in radiation of different tumors. Osteopontin (OPN) is a plasma protein that is related with tumor hypoxia. Furthermore, it is described that OPN expression is associated 
Table I. Plating efficiencies and radiosensitivity parameters of tumor cells cultured under normoxic and hypoxic conditions, respectively.

\begin{tabular}{|c|c|c|c|c|c|c|}
\hline \multirow[b]{2}{*}{ Cell line } & \multicolumn{2}{|c|}{$\mathrm{PE}$} & \multicolumn{2}{|c|}{$\mathrm{SF} 2$} & \multicolumn{2}{|c|}{ D10 } \\
\hline & Normoxia (\%) & Hypoxia (\%) & Normoxia & Hypoxia & Normoxia & Hypoxia \\
\hline SW480 & 55.3 & 54.7 & 0.76 & 0.83 & 7.11 & 25.01 \\
\hline SW620 & 100.0 & 21.2 & 0.41 & 0.49 & 4.04 & 5.09 \\
\hline HCT116 & 40.7 & 7.9 & 0.40 & 0.45 & 4.14 & 5.83 \\
\hline HT29 & 68.0 & 30.3 & 0.83 & 0.89 & 8.87 & 19.18 \\
\hline
\end{tabular}

The SF2 and D10 values were derived from the LQ model of the colony survival data. PE, plating efficiencies; SF2, surviving fraction at 2 Gy. D10, radiation dose yielding $10 \%$ survival.
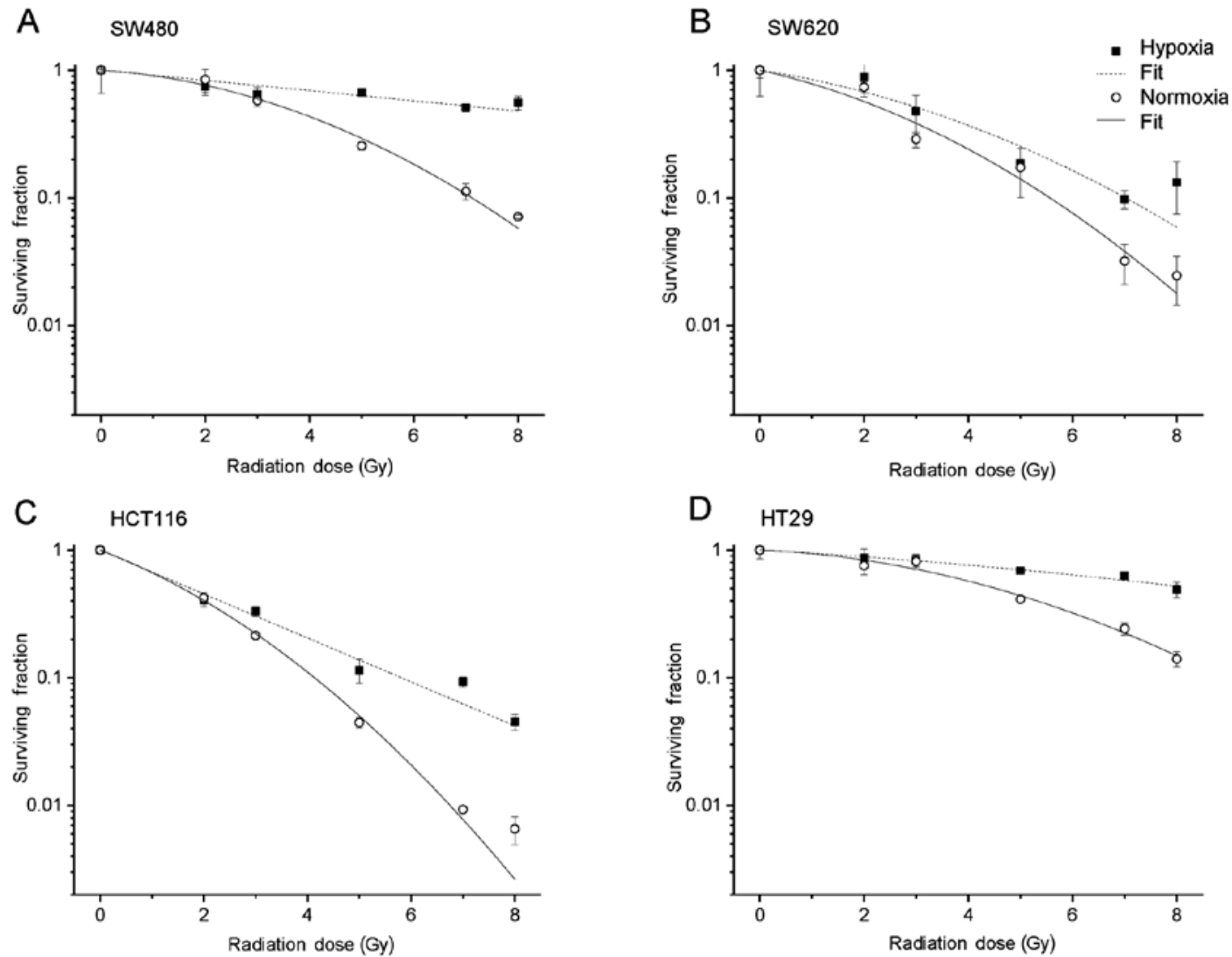

Figure 5. To investigate the clonogenic survival of the different colorectal carcinoma cell lines: (A) SW480, (B) SW620, (C) HCT116 and (D) HT29 by irradiation, cells were exposed to single doses ranging between 2 and $8 \mathrm{~Gy}$ or left untreated. Afterwards they were cultured under normoxic (empty circles) or hypoxic (filled boxes) conditions for 2 weeks. Surviving colonies contained $>50$ cells. Curves are the best fits of the linear quadratic equation.

with tumors with a poor prognosis. This is also true for some of its binding partners, such as the cell-surface protein CD44 and its splice variants (particularly CD44v6), expressed in aggressive cancer types $(24,25)$. The expression of CD44 has been described in so-called tumor stem cells and is a proposed marker for tumor-initiating cells in colon cancer, one of the most common cancers worldwide (26). Recently it was shown that the downregulation of CD44 expression on stem cells using siRNA or shRNA suppresses metastasis in breast cancer cells and makes the stem cells more sensitive to drug treatment $(27,28)$. In in vitro experiments with stem celllike brain tumor cells expressing only large amounts of the isoform CD44v6, cell growth was inhibited by downregulation of the receptor (knockdown). Furthermore, it was detected that interaction of OPN and CD44v6 caused an increase in phosphorylated AKT, suggesting the involvement of the AKT signaling pathway in signal transduction (29).

In our experiments, we investigated whether, and to what extent the cultivation of the cells under hypoxia affected the expression of OPN and its receptors CD44s and particularly CD44v6. Four different colon cancer cell lines were used: HT29, SW480 (derived from a primary tumor), SW620 (metastasis from the lymph node of the same patient) and HCT116. 
As expected, all tested cell lines had a more or less strong increase in OPN protein under hypoxic conditions (Figs. 2 and 4). These results agreed with the enhanced OPN mRNA expression noted in the SW480 and SW620 cells under hypoxia (Fig. 1A). In both cell lines a concordant response to hypoxia with an increase in OPN and CD44s mRNA was detected (Fig. 1A and B). This could be a first hint that OPN upregulates its own receptor under these conditions. However, further functional tests must be conducted to confirm this hypothesis. The metastatic cell line SW620 showed a much weaker basal expression of OPN and CD44s protein than the primary tumor cells (SW480) (Fig. 2). Furthermore, there was nearly no increase in CD44 expression and only a small increase in OPN protein under hypoxia. This is consistent with published data showing CD44 as a metastasis suppressor in some forms of prostate (30) and in pancreatic cancer (31). While there was a decrease in CD44s expression with increasing malignancy in some prostate cancers, there was a complete loss of CD44s expression in progressive pancreatic cancer, due to alternative splicing of the CD44 pre-RNA. Therefore, we assume that the higher tumorigenic and metastatic potential of SW620 cells (in comparison to SW480) shown by Hewitt et al is one reason for the different expression of CD44s and OPN (32).

Our results are also in accordance with recently published data, showing that colon cancer cells widely differ in regards to the expression of cancer stem cell markers such as CD44 (33). The authors speculate that the decreased basal CD44s expression in SW620 cells can be explained by transition from SW480 to SW620 cells, leading to the expression of a differentially post-translational modified CD44 protein. This may explain the weak signal for CD44s expression in comparison to the strong CD44v6 signal detected in the western blot analysis. Additionally, no CD44s protein could be detected in supernatants of SW620 cells in contrast to SW480 cells, as shown by ELISA assay (Fig. 3A). This may also favor the thesis of posttranslationally modified CD44. The splice-variant CD44v6 was also not detectable in SW620 cell supernatants, but in supernatants of SW480 cells, where its amount was reduced under hypoxic conditions (data not shown and Fig. 3A and B).

HT29 cells responded to hypoxia with a distinct downregulation of CD44s and CD44v6 protein (Fig. 2) accompanied by a significant decrease in OPN mRNA (Fig. 1A) and only a weak increase in OPN protein (Fig. 2). In addition, less CD44s and CD44v6 protein was detected in supernatants of the HT29 cells, when cells were cultured under hypoxic conditions (Fig. 3A and B). Due to the above-cited results (30), hypoxia may have caused progressive malignancy in HT29 cells by downregulation of CD44v6, acting as a metastasis suppressor in this colon cancer cell line. The development of strong radioresistance under hypoxic culture conditions could support the speculation of progressive malignancy in HT29 cells, triggered by hypoxia (Fig. 5). Our speculation of progressive malignancy in this cell line is supported by Flatmark et al who showed the aggressive behavior of HT29 cells within a panel of 12 colorectal cancer cell lines which produced lymph node metastases with a very high frequency (34).

In contrast, HCT116 cells showed an increase in CD44s at the mRNA and protein level under hypoxia (Figs. 1B and 2). In parallel, expression of its binding partner OPN was elevated as expected (Fig. 2). However, this was not consistent with the PCR results, showing a decreased OPN transcription rate under hypoxic conditions (Fig. 1A). Similar, but apparently contradictory results concerning OPN upregulation at the protein level and downregulation at the mRNA level were observed in the HT29 cells (Figs. 2 and 1A). Hence, the reduction in transcriptional activity of OPN mRNA could be an effect of a possible negative feed-back loop due to intracellular accumulation of OPN protein, shown by staining (Fig. 4). This fact could also explain the absence of OPN in cell culture supernatants of all used cell lines, regardless of the culture conditions; although an increase in OPN protein was detected by western blot analysis (Fig. 2). These results are in line with the western blotting outcomes in terms of the amount of OPN protein and recently published data from our group (35).

We may further examine, whether and to what extent OPN has an effect on the malignancy of colorectal tumor cell lines by influencing the expression of HIF-1A and HIF-2A. Both hypoxia-inducible factors mediate the response of cells to hypoxia by regulating downstream target genes. However, the functional roles of the two HIF isoforms are completely different in colon cancer as shown by Imamura et al, using SW480 cells (36). In this cell line, induction of HIF-1A promoted cell growth, while induction of HIF-2A appeared to restrain it. Furthermore, the loss of HIF-2A expression (but not of HIF-1A) was found to be associated with advanced tumor stage. Yang et al showed a significant downregulation of HIF-1A expression in breast cancer upon the efficient knockdown of OPN, promoting the radiosensitivity of the cells (37). Furthermore, increased survival of ovarian cancer cells was promoted by OPN and mediated via induction of HIF-1A expression through the PI3-K/Akt pathway (38). Jiang et al demonstrated in bone marrow-derived mesenchymal stem cells (BMSCs) an induction of adipogenic differentiation under hypoxic conditions regulated by HIF-1A, while in contrast OPN mRNA was decreased in these non-malignant cells (39). Recently, Cheng et al showed for the first time an association between OPN and HIF-2A expression in chondrocytes. In this previous study, the expression of HIF-2A at the mRNA level was inhibited by OPN (40).

In addition, irradiation itself had nearly no influence on the examined parameters. This is in concordance with our results obtained in head and neck cancer and glioblastoma cell lines, where OPN expression was strongly modulated by hypoxia and only to a minor extent by irradiation (35). An unchanged CD44 expression profile after irradiation was also observed by another group in Ewing sarcoma cell lines (41). These results may be important for the use of radiation therapy in the clinical setting, showing no risk of upregulation of OPN and its ligands.

Taken together, the experiments and descriptive results in this study show that no common statement can be made concerning the influence of hypoxia on CD44 and CD44v6 expression in colorectal cancer cell lines. Each cell line behaved differently, probably according to its state of malignancy, as discussed. Regarding OPN, all cell lines had in common a more or less strong increase in its protein expression and the development of radioresistance under hypoxia. Our planned future projects should provide more information concerning how hypoxia affects the cells on a functional level. 


\section{References}

1. Wai PY and Kuo PC: Osteopontin: Regulation in tumor metastasis. Cancer Metastasis Rev 27: 103-118, 2008.

2. Petrik D, Lavori PW, Cao H, Zhu Y, Wong P, Christofferson E, Kaplan MJ, Pinto HA, Sutphin P, Koong AC, et al: Plasma osteopontin is an independent prognostic marker for head and neck cancers. J Clin Oncol 24: 5291-5297, 2006.

3. Mack PC, Redman MW, Chansky K, Williamson SK Farneth NC, Lara PN Jr, Franklin WA, Le QT, Crowley JJ and Gandara DR; SWOG: Lower osteopontin plasma levels are associated with superior outcomes in advanced non-small-cell lung cancer patients receiving platinum-based chemotherapy: SWOG Study S0003. J Clin Oncol 26: 4771-4776, 2008.

4. Overgaard J, Eriksen JG, Nordsmark M, Alsner J and Horsman MR; Danish Head and Neck Cancer Study Group: Plasma osteopontin, hypoxia, and response to the hypoxia sensitiser nimorazole in radiotherapy of head and neck cancer: Results from the DAHANCA 5 randomised double-blind placebo-controlled trial. Lancet Oncol 6: 757-764, 2005

5. Bache M, Kappler M, Wichmann H, Rot S, Hahnel A, Greither T, Said HM, Kotzsch M, Würl P, Taubert H, et al: Elevated tumor and serum levels of the hypoxia-associated protein osteopontin are associated with prognosis for soft tissue sarcoma patients. BMC Cancer 10: 132, 2010

6. Le QT, Sutphin PD, Raychaudhuri S, Yu SC, Terris DJ, Lin HS Lum B, Pinto HA, Koong AC and Giaccia AJ: Identification of osteopontin as a prognostic plasma marker for head and neck squamous cell carcinomas. Clin Cancer Res 9: 59-67, 2003.

7. Nordsmark M, Bentzen SM, Rudat V, Brizel D, Lartigau E, Stadler P, Becker A, Adam M, Molls M, Dunst J, et al: Prognostic value of tumor oxygenation in 397 head and neck tumors after primary radiation therapy. An international multi-center study. Radiother Oncol 77: 18-24, 2005.

8. Hockel M, Schlenger K, Aral B, Mitze M, Schaffer U and Vaupel P: Association between tumor hypoxia and malignant progression in advanced cancer of the uterine cervix. Cancer Res 56: 4509-4515, 1996.

9. Nordsmark M, Alsner J, Keller J, Nielsen OS, Jensen OM, Horsman MR and Overgaard J: Hypoxia in human soft tissue sarcomas: Adverse impact on survival and no association with p53 mutations. Br J Cancer 84: 1070-1075, 2001

10. Polat B, Wohlleben G, Katzer A, Djuzenova CS, Technau A and Flentje M: Influence of osteopontin silencing on survival and migration of lung cancer cells. Strahlenther Onkol 189: 62-67, 2013.

11. Hahnel A, Wichmann H, Kappler M, Kotzsch M, Vordermark D, Taubert $\mathrm{H}$ and Bache $\mathrm{M}$ : Effects of osteopontin inhibition on radiosensitivity of MDA-MB-231 breast cancer cells. Radiat Oncol 5: 82, 2010.

12. Hahne JC, Meyer SR, Kranke P, Dietl J, Guckenberger M, Polat B and Hönig A: Studies on the role of osteopontin-1 in endometrial cancer cell lines. Strahlenther Onkol 189: 1040-1048, 2013.

13. Zöller M: CD44: Can a cancer-initiating cell profit from an abundantly expressed molecule? Nat Rev Cancer 11: 254-267, 2011.

14. Marroquin CE, Downey L, Guo H and Kuo PC: Osteopontin increases CD44 expression and cell adhesion in RAW 264.7 murine leukemia cells. Immunol Lett 95: 109-112, 2004.

15. Xu Y, Stamenkovic I and Yu Q: CD44 attenuates activation of the hippo signaling pathway and is a prime therapeutic target for glioblastoma. Cancer Res 70: 2455-2464, 2010.

16. Denhardt DT, Giachelli CM and Rittling SR: Role of osteopontin in cellular signaling and toxicant injury. Annu Rev Pharmacol Toxicol 41: 723-749, 2001.

17. Khan SA, Cook AC, Kappil M, Günthert U, Chambers AF, Tuck AB and Denhardt DT: Enhanced cell surface CD44 variant (v6, v9) expression by osteopontin in breast cancer epithelial cells facilitates tumor cell migration: Novel post-transcriptional, post-translational regulation. Clin Exp Metastasis 22: 663-673, 2005.

18. Christofori G: Changing neighbours, changing behaviour: Cell adhesion molecule-mediated signalling during tumour progression. EMBO J 22: 2318-2323, 2003.

19. Shi J, Zhou Z, Di W and Li N: Correlation of CD44v6 expression with ovarian cancer progression and recurrence. BMC Cancer 13: $182,2013$.

20. Xie JW, Chen PC, Zheng CH, Li P, Wang JB, Lin JX, Lu J, Chen QY, Cao LL, Lin M, et al: Evaluation of the prognostic value and functional roles of CD44v6 in gastric cancer. J Cancer Res Clin Oncol 141: 1809-1817, 2015.
21. Chen P, Huang HF, Lu R, Wu Y and Chen YZ: Prognostic significance of $\mathrm{CD} 44 \mathrm{v} 6 / \mathrm{v} 7$ in acute promyelocytic leukemia. Asian Pac J Cancer Prev 13: 3791-3794, 2012

22. Yokota A, Ishii G, Sugaya Y, Nishimura M, Saito Y and Harigaya K: Expression of exon v6-containing CD44 isoforms is related to poor prognosis of acute myelocytic leukemia. Hematol Oncol 16: 131-141, 1998.

23. Livak KJ and Schmittgen TD: Analysis of relative gene expression data using real-time quantitative PCR and the $2^{-\Delta \Delta C \mathrm{~T}}$ method. Methods 25: 402-408, 2001.

24. Naor D, Nedvetzki S, Golan I, Melnik L and Faitelson Y: CD44 in cancer. Crit Rev Clin Lab Sci 39: 527-579, 2002.

25. Günthert U: CD44 in malignant disorders. Curr Top Microbiol Immunol 213: 271-285, 1996.

26. Huh JW, Kim HR, Kim YJ, Lee JH, Park YS, Cho SH and Joo JK: Expression of standard CD44 in human colorectal carcinoma: Association with prognosis. Pathol Int 59: 241-246, 2009.

27. Fang XJ, Jiang H, Zhao XP and Jiang WM: The role of a new CD44st in increasing the invasion capability of the human breast cancer cell line MCF-7. BMC Cancer 11: 290, 2011.

28. Van Phuc P, Nhan PL, Nhung TH, Tam NT, Hoang NM, Tue VG, Thuy DT and Ngoc PK: Downregulation of CD44 reduces doxorubicin resistance of $\mathrm{CD} 44^{+} \mathrm{CD} 24^{-}$breast cancer cells. Onco Targets Ther 4: 71-78, 2011.

29. Jijiwa M, Demir H, Gupta S, Leung C, Joshi K, Orozco N, Huang T, Yildiz VO, Shibahara I, de Jesus JA, et al: CD44v6 regulates growth of brain tumor stem cells partially through the AKT-mediated pathway. PLoS One 6: e24217, 2011.

30. Gao AC, Lou W, Dong JT and Isaacs JT: CD44 is a metastasis suppressor gene for prostatic cancer located on human chromosome 11p13. Cancer Res 57: 846-849, 1997.

31. Abetamann V, Kern HF and Elsässer HP: Differential expression of the hyaluronan receptors CD44 and RHAMM in human pancreatic cancer cells. Clin Cancer Res 2: 1607-1618, 1996.

32. Hewitt RE, McMarlin A, Kleiner D, Wersto R, Martin P, Tsokos M, Stamp GW and Stetler-Stevenson WG: Validation of a model of colon cancer progression. J Pathol 192: 446-454, 2000.

33. Wang C, Xie J, Guo J, Manning HC, Gore JC and Guo N: Evaluation of CD44 and CD133 as cancer stem cell markers for colorectal cancer. Oncol Rep 28: 1301-1308, 2012.

34. Flatmark K, Maelandsmo GM, Martinsen M, Rasmussen H and Fodstad Ø: Twelve colorectal cancer cell lines exhibit highly variable growth and metastatic capacities in an orthotopic model in nude mice. Eur J Cancer 40: 1593-1598, 2004.

35. Wohlleben G, Scherzad A, Güttler A, Vordermark D, Kuger S, Flentje $\mathrm{M}$ and Polat B: Influence of hypoxia and irradiation on osteopontin expression in head and neck cancer and glioblastoma cell lines. Radiat Oncol 10: 167, 2015.

36. Imamura T, Kikuchi H, Herraiz MT, Park DY, Mizukami Y, Mino-Kenduson M, Lynch MP, Rueda BR, Benita Y, Xavier RJ, et al: HIF-1alpha and HIF-2alpha have divergent roles in colon cancer. Int J Cancer 124: 763-771, 2009.

37. Yang L, Zhao W, Zuo WS, Wei L, Song XR, Wang XW, Zheng G and Zheng MZ: Silencing of osteopontin promotes the radiosensitivity of breast cancer cells by reducing the expression of hypoxia inducible factor 1 and vascular endothelial growth factor. Chin Med J 125: 293-299, 2012.

38. Song G, Cai QF, Mao YB, Ming YL, Bao SD and Ouyang GL: Osteopontin promotes ovarian cancer progression and cell survival and increases HIF-1alpha expression through the PI3-K/Akt pathway. Cancer Sci 99: 1901-1907, 2008.

39. Jiang C, Sun J, Dai Y, Cao P, Zhang L, Peng S, Zhou Y, Li G, Tang $\mathrm{J}$ and Xiang J: HIF-1 A and C/EBPs transcriptionally regulate adipogenic differentiation of bone marrow-derived MSCs in hypoxia. Stem Cell Res Ther 6: 21,2015.

40. Cheng C, Zhang FJ, Tian J, Tu M, Xiong YL, Luo W, Li YS, Song BB, Gao SG and Lei GH: Osteopontin inhibits HIF-2 $\alpha$ mRNA expression in osteoarthritic chondrocytes. Exp Ther Med 9: 2415-2419, 2015

41. Könemann S, Malath J, Bölling T, Kolkmeyer A, Janke K, Riesenbeck D, Hesselmann S, Nguyen TP, Diallo R, Vormoor J, et al: Changed adhesion molecule profile of Ewing tumor cell lines and xenografts under the influence of ionizing radiation. Anticancer Res 24: 1637-1644, 2004. 